\title{
GENERAL DISCUSSION AND SHORT CONTRIBUTION
}

\section{The Influences of Bulk Viscosity on the Existence of the Initial Singularity}

M. Heller and L. Suszycki : In previous work (Heller et al., 1973; Heller and Suszycki, 1973) bulk viscosity was introduced into the framework of Friedmann-Lemaitre cosmological models under the highly idealized assumption of constant coefficient of bulk viscosity. The Einstein's equations with the Robertson-Walker metric and the energy-momentum tensor as given, for example, by Landau and Lifshitz (1959) or Weinberg (1971) are :

$$
\begin{aligned}
& \kappa \varrho c^{2}=-\Lambda+3 \frac{k c^{2}+\dot{R}^{2}}{c^{2} R^{2}} \\
& \kappa p=\Lambda-\frac{2 R \ddot{R}+\dot{R}^{2}+k c^{2}}{c^{2} R^{2}}+3 \kappa \xi \frac{\dot{R}}{R},
\end{aligned}
$$

where $\xi=$ const is the coefficient of bulk viscosity. The shear viscosity term vanishes on account of isotropy.

Our main results for the dust filled models are the following:

(1) The introduction of the bulk viscosity removes the initial singularity provided it is allowed by the Hawking-Penrose theorem;

(2) Many models obtained with this method, although analytically regular, have regions with negative density.

As a next step we have assumed a linear dependence of the bulk viscosity coefficient on density:

$$
\xi=\frac{2 \alpha}{3 \kappa} \varrho
$$

where $\alpha=$ const. The numerical calculations for flat, closed and open world models

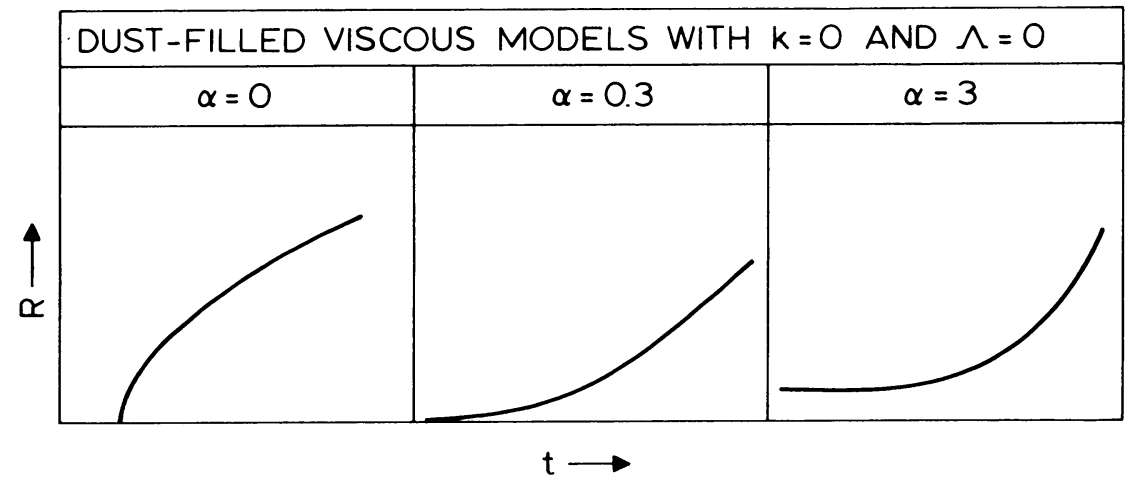

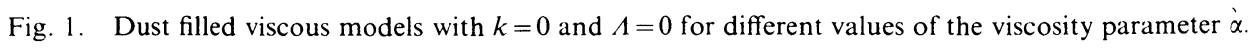


filled with dust or radiation were performed. The preliminary results seem to be interesting:

(1) There are no models with negative density;

(2) There are no viscous flat models with an initial singularity. Therefore, in these cases the bulk viscosity appears to be an effective mechanism for removing the singularity.

In cases in which the singularity is removed the energy condition of the HawkingPenrose theorem is violated.

An example of the removal of the singularity is shown in Figure 1.

More detailed results and their discussion will be published.

\section{References}

Heller, M. and Suszycki, L.: 1973, Acta Phys. Pol., in press.

Heller, M., Klimek, Z., and Suszycki, L.: 1973, Astrophys. Space Sci. 20, 205.

Landau, L. and Lifshitz, E. M.: 1959, Fluid Mechanics, London.

Weinberg, S.: 1971, Astrophys. J. 168, 175.

Grishchuk: Do you suppose that the viscous terms appearing in your energymomentum tensor are small with respect to the perfect fluid terms?

Heller: No. In our calculations viscous terms can be large and our considerations are therefore of mathematical rather than of physical significance.

Stewart: For a gas with particles of mass $m$ bulk viscosity is largest when $m c^{2} / k T \sim$ 1. Even then in most cases it is at least $10^{-3}$ times smaller than shear viscosity, and in fact vanishes in the non-relativistic and ultra-relativistic limits. Thus it is unlikely to influence cosmological models in the way you describe. In other words your assumptions that the viscosity coefficient is a constant or proportional to density are unrealistic.

Heller: I agree - what is in agreement with the Hawking-Penrose theorem must not be in agreement with physics.

Westervelt: Do you consider the increase in the isotropic part of the stress-energy tensor caused by dissipation caused by the bulk viscosity?

Heller: Yes.

Wheeler: We have heard from Hawking of the very special limitations on inhomogeneity which he argues to be necessary if the Universe is to permit life as we know it. We have to add these considerations to related considerations of Carter and of Dicke. Dicke reasons that a universe of lesser size than this would live a lesser time and would not give opportunity for thermonuclear combusion to produce heavy elements and life and awareness of the Universe. In effect Dicke invites us to consider the proposition that "the Universe is as big as it is because we are here." Carter proposes a similar thesis, that the constants of physics have the values they do because other values would exclude life. But a few percent change in the fine structure constant one way would require all stars to be red stars and no star like the Sun would be possible. A 
few percent change the other way would make all stars blue stars and again no star like the Sun would be possible. The considerations of Hawking, Dicke and Carter raise the question whether man is involved in the design of the Universe in a much more central way than one can previously imagine. This issue is so interesting that it would be good to hear a bit more about it from Brandon Carter himself who is here with us now. 